\title{
Solar-like oscillations in the metal-poor subgiant $v$ Indi
}

\section{Acoustic spectrum and mode lifetime}

\author{
F. Carrier ${ }^{1,2}$, H. Kjeldsen ${ }^{3}$, T. R. Bedding ${ }^{4}$, B. J. Brewer ${ }^{4}$, R. P. Butler ${ }^{5}$, P. Eggenberger ${ }^{2,6}$, \\ F. Grundahl ${ }^{3}$, C. McCarthy ${ }^{5}$, A. Retter ${ }^{4,7}$, and C. G. Tinney ${ }^{8}$
}

1 Instituut voor Sterrenkunde, Katholieke Universiteit Leuven, Celestijnenlaan 200D, 3001 Leuven, Belgium e-mail: fabien@ster. kuleuven.be

2 Observatoire de Genève, Université de Genève, 51 chemin des Maillettes, 1290 Sauverny, Switzerland

3 Department of Physics and Astronomy, University of Aarhus, 8000 Aarhus C, Denmark e-mail: [hans; fgj; tbn] @phys.au.dk

4 School of Physics A28, University of Sydney, NSW 2006, Australia e-mail: [bedding; brewer] aphysics.usyd.edu.au

5 Carnegie Institution of Washington, Department of Terrestrial Magnetism, 5241 Broad Branch Road NW, Washington, DC 20015-1305, USA e-mail: [paul;chris]@dtm.ciw.edu

${ }^{6}$ Institut d'Astrophysique et de Géophysique, Université de Liège, 17 allée du 6 Août, Bât. B5c, 4000 Liège, Belgium e-mail: eggenberger@astro.ulg.ac.be

7 Department of Astronomy and Astrophysics, PennSylvania State University, 525 Davey Lab, University Park, PA 16802-6305, USA e-mail: retter@astro.psu.edu

8 Anglo-Australian Observatory, PO Box 296, Epping, NSW 1710, Australia e-mail: cgt@aaoepp.aao.gov.au

Received 8 December 2006 / Accepted 18 May 2007

\section{ABSTRACT}

\begin{abstract}
Context. Convection in stars excites resonant acoustic waves which depend on the sound speed inside the star, which in turn depends on properties of the stellar interior. Therefore, asteroseismology is an unrivaled method to probe the internal structure of a star. Aims. We made a seismic study of the metal-poor subgiant star $v$ Indi with the goal of constraining its interior structure.

Methods. Our study is based on a time series of 1201 radial velocity measurements spread over 14 nights obtained from two sites, Siding Spring Observatory in Australia and ESO La Silla Observatory in Chile.

Results. The power spectrum of the high precision velocity time series clearly presents several identifiable peaks between 200 and $500 \mu \mathrm{Hz}$ showing regularity with a large and small spacing of $\Delta v=25.14 \pm 0.09 \mu \mathrm{Hz}$ and $\delta v_{02}=2.96 \pm 0.22 \mu \mathrm{Hz}$ at $330 \mu \mathrm{Hz}$. Thirteen individual modes have been identified with amplitudes in the range 53 to $173 \mathrm{~cm} \mathrm{~s}^{-1}$. The mode damping time is estimated to be about 16 days (1- $\sigma$ range between 9 and 50 days), substantially longer than in other stars like the Sun, the $\alpha$ Cen system or the giant $\xi$ Hya.
\end{abstract}

Key words. stars: individual: $v$ Indi - stars: oscillations - stars: variables: general - stars: interiors - stars: late-type

\section{Introduction}

The analysis of the oscillation spectrum provides an unrivaled method to probe the stellar internal structure. The frequencies of these oscillations depend on the sound speed inside the star, which in turn depends on density, temperature, gas motion and other properties of the stellar interior. High-precision spectrographs have led in recent years to a rapidly growing list of solarlike oscillation detections.

The recent achievements are reviewed by Bedding \& Kjeldsen $(2006,2007)$. All of the previous detections of oscillations were made on stars with metallicities close to solar. We recently reported observations of the metal-poor subgiant star $v$ Indi made in both Chile with the spectrograph CORALIE and in Australia with UCLES (Bedding et al. 2006, hereafter Paper I). These observations covered a 14 nights time span and had the benefit of two-site coverage.

In Paper I, we described the radial velocity time series and calculated the corresponding power spectrum weighted by the measurement uncertainties. We reached a noise floor of $14.9 \mathrm{~cm} \mathrm{~s}^{-1}$ in the combined amplitude spectrum, which is quite high compared to other studies with the same instruments (see e.g. Bouchy \& Carrier 2002; and Bedding et al. 2004). This difference can be explained by the extremely low metallicity (about $3 \%$ of solar) and the faint magnitude $\left(m_{V}=5.28\right)$ of $v$ Indi. Oscillation modes are present around $0.32 \mathrm{mHz}$ with a maximal amplitude per mode of $95 \mathrm{~cm} \mathrm{~s}^{-1}$. A large frequency separation was determined by autocorrelation and Bayesian methods to be $24.25 \pm 0.25 \mu \mathrm{Hz}$.

In this paper, we will extend the analysis by extracting individual oscillation frequencies, by measuring the large and small separations, and by estimating the mode lifetimes.

\section{Frequency analysis}

The power spectrum of the time series, shown in Fig. 1, exhibits a series of peaks between 200 and $550 \mu \mathrm{Hz}$. In solar-like stars, p-mode oscillations are expected to produce a characteristic 


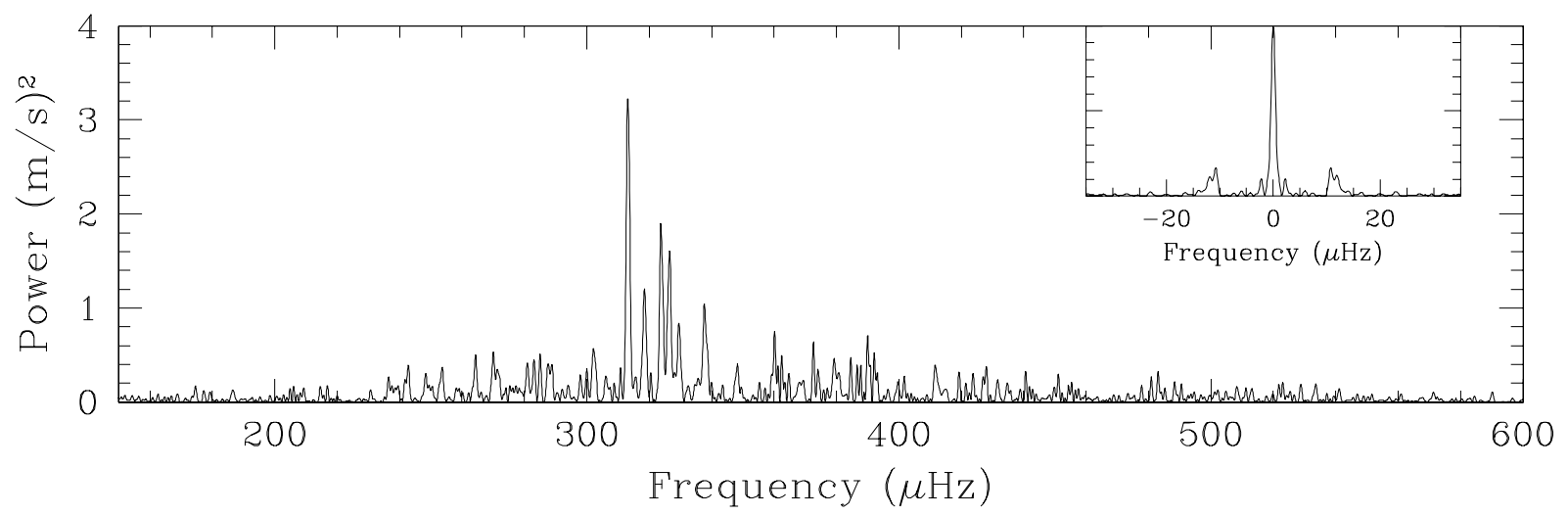

Fig. 1. Power spectrum of the radial velocity measurements of $v$ Indi. The inset shows the spectral window.

comb-like structure in the power spectrum with mode frequencies $v_{n, \ell}$ reasonably well approximated by the asymptotic relation (Tassoul 1980):

$v_{n, \ell} \approx \Delta v\left(n+\frac{\ell}{2}+\epsilon\right)-\ell(\ell+1) D_{0}$.

Here $D_{0}$ (which equals $\frac{1}{6} \delta v_{02}$ if the asymptotic relation holds exactly) and $\epsilon$ are sensitive to the sound speed near the core and in the surface layers, respectively. The quantum numbers $n$ and $\ell$ correspond to the radial order and the angular degree of the modes, and $\Delta v$ and $\delta v_{02}$ are the large and small spacings. To fit to this relation, the large separation was measured in Paper I by autocorrelation and Bayesian methods and was found to be $24.25 \pm 0.25 \mu \mathrm{Hz}$.

Note that a subgiant such as $v$ Indi is expected to show substantial deviations from the regular comb-like structure described above. This is because some mode frequencies, except for $\ell=0$, could be shifted from their usual regular spacing by avoided crossings with gravity modes in the stellar core (also called "mode bumping") (see e.g. Christensen-Dalsgaard et al. 1995 and Fernandes \& Monteiro 2003). We must keep in mind the possibility of these mixed modes when attempting to identify oscillation modes in the power spectrum.

The next step is to measure the frequencies of the strongest peaks in the power spectrum. The frequencies were extracted using an iterative algorithm which identifies the highest peak between 150 and $600 \mu \mathrm{Hz}$ and subtracts it from the time series (see e.g. Carrier et al. 2003; and Kjeldsen et al. 2005). In the present analysis we are only marginally affected by the window sidebands compared to mono-site observations analysis. The highest amplitude modes are therefore expected to be located at their correct position and they do not need to be shifted by the daily aliases (Kjeldsen et al. 2003; Carrier et al. 2005a,b). In total, 27 frequencies were extracted having an amplitude 3 times greater than the noise. These amplitudes are measured from the best-fitting sinusoid, whereas the noise is computed as the mean in the amplitude spectrum, which is the square root of the power spectrum, at high frequencies. Note that the noise level at high frequencies $(600-1000 \mu \mathrm{Hz})$ has a value of $14.9 \mathrm{~cm} \mathrm{~s}^{-1}$ (Paper I) and is assumed constant on the whole range $150-600 \mu \mathrm{Hz}$.

All frequencies with a signal-to-noise ratio $(S N R)$ above 3 were then placed in an echelle diagram, without shifting any of them, constructed with the value for the large separation found in Paper I (see Fig. 2 left). This diagram does not seem to show a classical asymptotic structure, since only one vertical ridge can be seen (dotted line at $22 \mu \mathrm{Hz}$ ). A possible way to explain such a graphic is to associate the ridge at $22 \mu \mathrm{Hz}$ to $\ell=2$ modes and

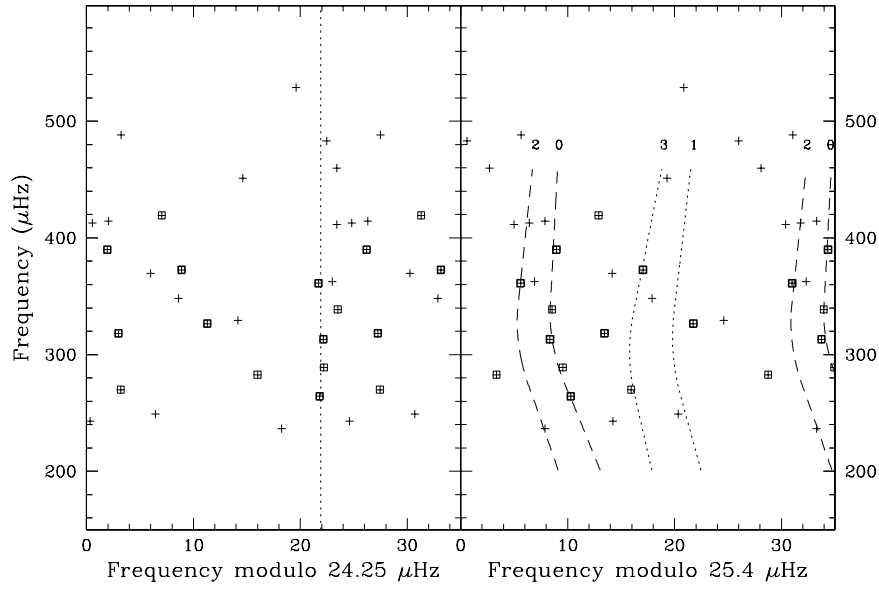

Fig. 2. Echelle diagrams for two different values of the large separation. The modes with a $S N R$ greater than 4 are indicated with a square, and the ones with a $S N R$ greater than 5 are indicated in boldface. Left: echelle diagram where $24.25 \mu \mathrm{Hz}$ is used as a value for the large separation (see Paper I). Right: echelle diagram with a large separation of $25.4 \mu \mathrm{Hz}$. The lines are just meant as a "guide".

the quasi-line near $27 \mu \mathrm{Hz}$ to $\ell=0$ modes. This identification is not very convincing and would imply a small separation of $5 \mu \mathrm{Hz}$, which is too large for this kind of star.

Another way to understand this diagram is to increase slightly the large spacing and allow curvature along the frequency axis (see Fig. 2 right). Indeed, large separation determination methods based on the asymptotic relation (as the autocorrelation presented in Paper I), do not take into account any curvature. With curvature, there is no correct value for the large spacing, it is a function of frequency. With a large separation of $25.4 \mu \mathrm{Hz}$, as in the right panel, we can identify ridges corresponding to $\ell=0$ and $\ell=2$. These are marked with dashed lines.

With the ridges for $\ell=0$ and 2 marked as shown, we then used the asymptotic relation to calculate the expected positions for $\ell=1$ and 3 . These are shown by dotted lines in the right panel of Fig. 2. We can see that two strong peaks lie exactly on the line for $\ell=3$, giving us some confidence that we have found the correct solution. We also see an absence of peaks on the $\ell=1$ line and a few strong peaks that do not lie on any of the lines. This is consistent with the presence of mixed $\ell=1$ modes that are shifted by avoided crossings, as described above.

Based in Fig. 2, several modes were identified in $v$ Indi. The frequency, amplitude, $S N R$ of these modes are given in Table 1 
Table 1. Amplitude and frequency for individual oscillation modes and their identification. The mean noise in the amplitude spectrum is $11.9 \mathrm{~cm} \mathrm{~s}^{-1}$.

\begin{tabular}{cccc}
\hline $\begin{array}{c}\text { Frequency } \\
{[\mu \mathrm{Hz}]}\end{array}$ & $\begin{array}{c}\text { Amplitude } \\
\mathrm{cm} \mathrm{s}^{-1}\end{array}$ & $\begin{array}{c}S N R \\
313.1\end{array}$ & $\begin{array}{c}\text { Mode ID } \\
\ell\end{array}$ \\
\hline 372.6 & 108 & 11.6 & 0 \\
361.3 & 105 & 7.2 & $3 ?$ \\
318.2 & 103 & 6.9 & 2 \\
389.9 & 100 & 6.7 & 0 \\
326.5 & 97 & 6.5 & 1 \\
264.3 & 91 & 6.1 & 0 \\
338.8 & 89 & 6.0 & 0 \\
282.7 & 73 & 4.9 & $1 ?$ \\
288.9 & 69 & 4.6 & 0 \\
269.9 & 69 & 4.6 & $3 ?$ \\
236.5 & 54 & 3.6 & 2 \\
412.8 & 54 & 3.6 & 2 \\
\hline
\end{tabular}

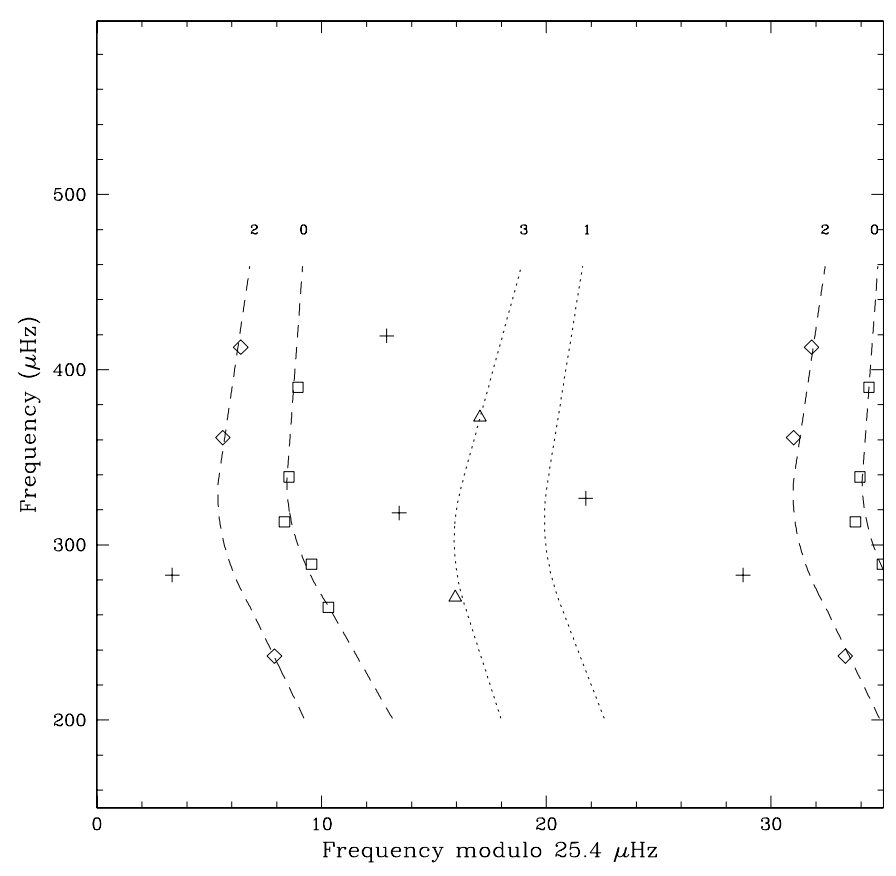

Fig. 3. Echelle diagram for the frequencies given in Table 1 and 2. The lines are just meant as a "guide". Squares, crosses, rhombuses and triangles represent the $\ell=0,1,2$ and 3 modes respectively.

with also the identified degree. Since the amplitude of the modes in an iterative fitting algorithm depends slightly on the selection order, a least squares fit was applied at the end to determine all amplitudes simultaneously. We retained all peaks with a $S N R$ greater than 4 . We also added two weaker peaks that were in good agreement with the $\ell=2$ ridge (see Table 1 ). It is expected in evolved stars that $\ell=1$ modes are the first to be mixed modes and do not follow the asymptotic relation. Thus, all large peaks which can not be determined as $\ell=0,2$ or 3 are listed as $\ell=$ 1 modes. $\ell=3$ modes are also less reliable. Finally, all the selected modes are shown in the echelle diagram in Fig. 3 and listed in Table 2.

Based in Fig. 3 and on the frequencies given in Table 2, the large and small separation are deduced taking into account only the $\ell=0$ and 2 modes, as $\ell=1$ modes do not follow the asymptotic relation and $\ell=3$ ones do not have a secure identification. The large separation has a value of $25.14 \pm 0.14 \mu \mathrm{Hz}$ at $0.33 \mathrm{mHz}$ which corresponds to the weighted average of individual values
Table 2. Frequency (in $\mu \mathrm{Hz}$ ) and mode identification for individual oscillation modes. The errors on the frequencies are given in brackets $\left(10^{-1} \mu \mathrm{Hz}\right)$ according simulations described in Sect. 3.3. The identification of frequencies in brackets is less secure.

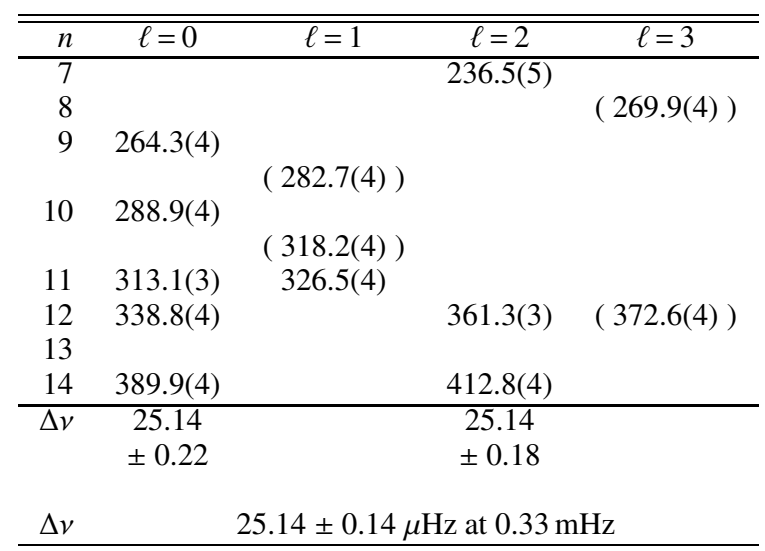

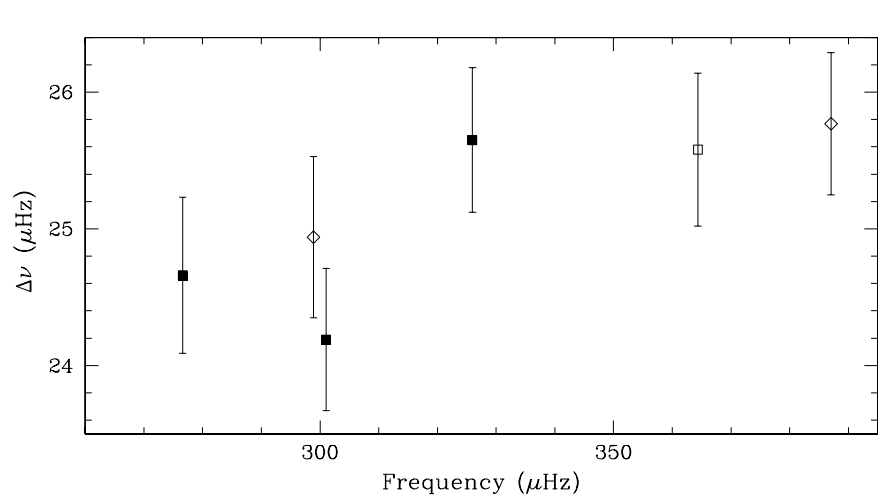

Fig. 4. Large spacing $\Delta v$ versus frequency for p-modes of degree $\ell=0$ $(\square)$ and $\ell=2(\diamond)$. Open symbols correspond to large spacing averages taken between non-successive modes.

given for each degree (see Table 2). The variation of the large separation versus frequency is presented in Fig. 4.

For the small separation, there are no pairs of adjacent $\ell=$ 0,2 modes, and so missing $\ell=0$ modes were linearly interpolated. We find a value of $\delta v_{02}=3.0 \pm 0.3 \mu \mathrm{Hz}$ at $0.33 \mathrm{mHz}$. It is not possible to calculate a reliable value for $\delta v_{13}$ since $\ell=1$ modes do not at all follow the asymptotic relation, and since the identification of $\ell=3$ modes could be in error. In fact, one could also identify the $\ell=3$ as $\ell=1$ modes. This fact should be remembered when comparing the observed and theoretical frequencies.

\section{Mode lifetimes}

We have used two methods to estimate the mode lifetimes from our data. The first uses the distribution of mode amplitudes and the second uses the scatter of the frequencies about the ridges in the echelle diagram. In both cases, we calibrated the results using simulations.

\subsection{Mode lifetimes by amplitude comparison}

The idea is to compare the amplitudes of peaks in the power spectrum with simulations that have the same amplitude envelope as the observations and a range of mode lifetimes. The simulator for generating solar-like oscillation time series is similar to that described by De Ridder et al. (2006). For the input 


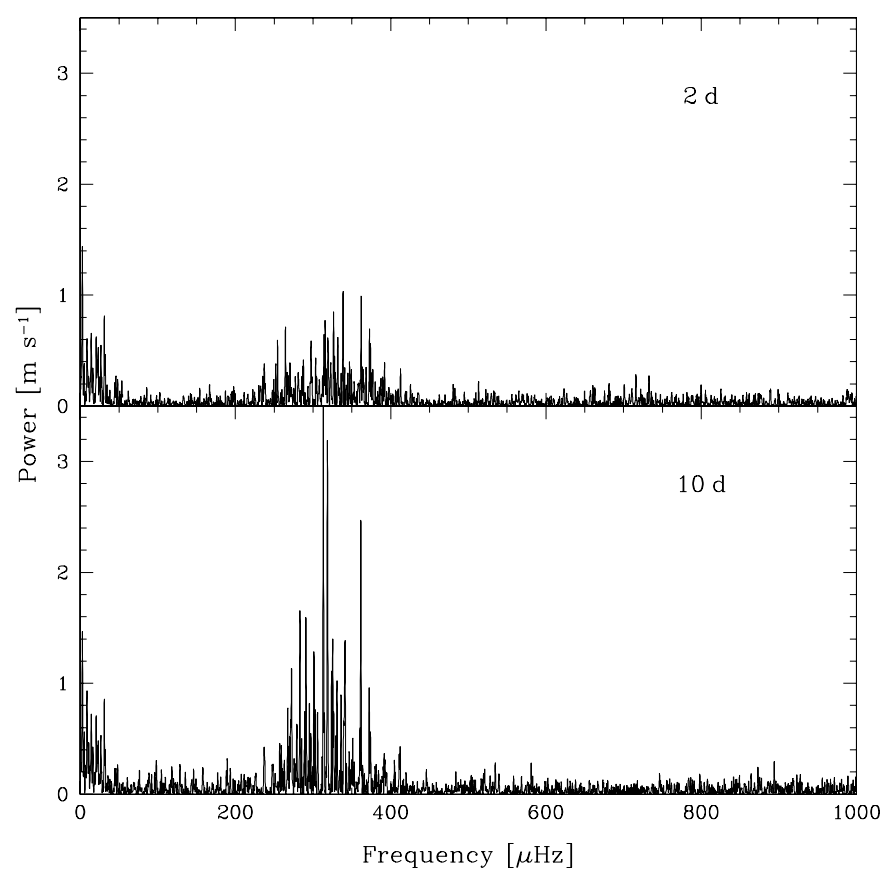

Fig. 5. Examples of two simulations of the $v$ Indi time series using mode lifetimes of 2 days (top) and 10 day (bottom). Higher peaks are in general found for the longer lifetime.

amplitudes, we used the smoothed amplitude spectrum show in Fig. 7 of Paper I. We ran ten simulations for each of six mode lifetimes $(1,2,5,10,20$ and 50 days). Two examples, with mode lifetimes of $2 \mathrm{~d}$ and $10 \mathrm{~d}$, are shown in Fig. 5. We see a significant difference between them, which can be used to estimate the mode lifetime.

In order to do this, we extracted the 7 highest peaks from each simulated power spectrum (using iterative fitting) and compared their amplitudes with those extracted from the observed series. As a simple measure of the degree of agreement, we adopted the following method. For a given simulation, we compared the highest peak with the highest peak in the observation. We then compared the second highest simulated peak with the second highest observed peak, and so on. The fraction of peaks in the simulation that were above the observed ones, in this pairwise comparison, gave the measure of agreement, with $50 \%$ indicating the best agreement.

The results are shown in Fig. 6, which shows the fraction of simulated peaks that were above the observations. We can read off the most likely value of the mode lifetime, corresponding to a fraction of 0.5 , to be 17 days. However, this measurement is not very precise. The dashed curve is the cumulative distribution of a normal distribution, and we see that the one- $\sigma$ range for the mode lifetime is 2 to 141 days, or in the logarithmic units:

$\log _{10}(\tau / d)=1.23 \pm 0.92$

\subsection{Mode lifetime by frequency scatter}

The other way to extract information on mode lifetimes is to examine the scatter of measured frequencies about the ridges in the echelle diagram. For these observations, this is only possible for the five $\ell=0$ modes. If the modes were coherent (i.e. pure sinusoids with infinite lifetimes), we would be able to measure them with a typical accuracy of $0.1 \mu \mathrm{Hz}$. The observed scatter is much

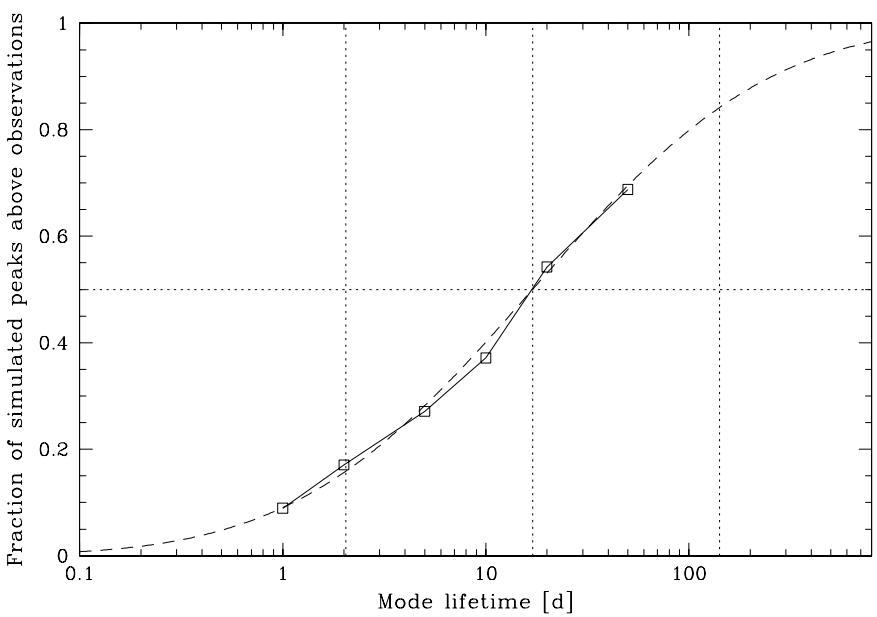

Fig. 6. Calibration of the simulations to determine mode lifetime from the peak amplitudes. It shows the fraction of modes in the simulations that have amplitudes above the observed mode amplitudes. The dashed curve is a normally distributed function that presents the best fit. Vertical dashed lines show the resulting mode lifetime and the values at $\pm 1 \sigma$.

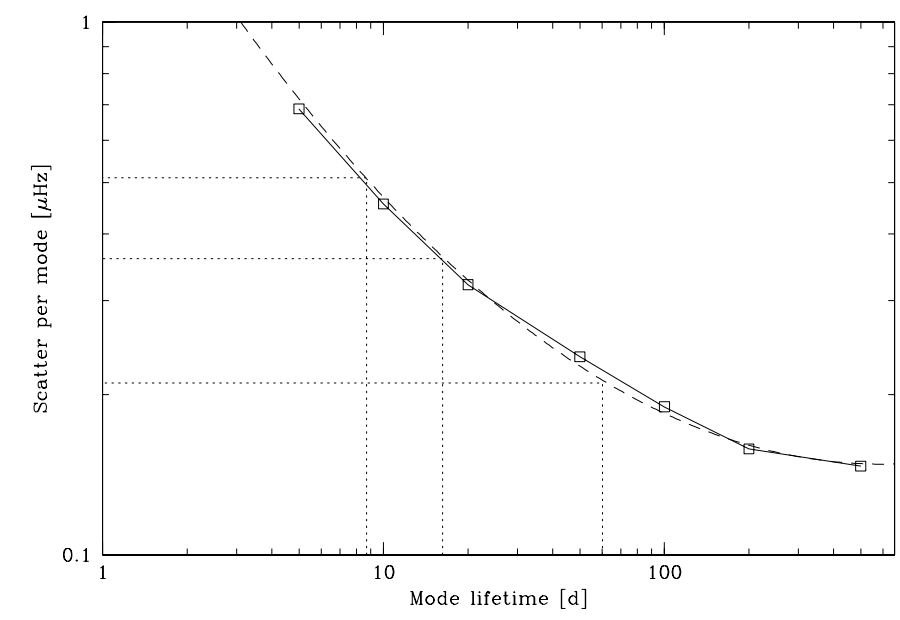

Fig. 7. Relation between scatter per mode and mode lifetime in days. The curve is a fit to points that came out of 700 individual simulated time series data. The mode lifetime corresponding to the best fitted frequency scatter is shown together with values for $\pm 1 \sigma$.

greater than this, which we interpret, at least for $\ell=0$ modes, as reflecting the finite lifetimes of the oscillation modes.

As is well established for the Sun, the power spectrum of a stochastically excited oscillation that is observed for long enough will display a series of closely spaced peaks under a Lorentzian profile, the width of which indicates the mode lifetime (e.g. Toutain \& Fröhlich 1992). If the observations are not long enough to resolve the Lorentzian profile, then the effect of the finite lifetime is to randomly shift each oscillation peak from its true position by a small amount. This is responsible for the scatter of the frequencies.

The scatters are determined by assuming that peaks are unaffected by the rotation of the star and that the large spacing varies only slightly between three consecutive detected modes. For each measured $\ell=0$ frequency, except those at the ends of the ridges, we calculated the difference between the measured frequency and that expected from linear interpolating the positions of the two nearest neighbors. This quantity was then transformed to the rms scatter of that peak about its expected position, in order to easily compare it with simulations (see Kjeldsen et al. 2005). In this way, three values of scatter per mode could 
be calculated, which have a mean of $0.36 \mu \mathrm{Hz} \pm 0.15 \mu \mathrm{Hz}$, for an average observed amplitude of $95 \mathrm{~cm} \mathrm{~s}^{-1}$.

In order to calibrate the relation between frequency scatter and mode lifetime, we ran 700 simulations using the same sampling and statistical weighting as the real time series. The results are shown in Fig. 7 and the best fit corresponds to a mode lifetime of 16.2 days (between 8.7 and 60.8 days at $1 \sigma$ ). Expressed in logarithmic units, we get:

$\log _{10}(\tau / d)=1.21_{-0.27}^{+0.57}$.

Combining this measurement of mode lifetime with the previous one, and assuming that they are independent estimates, we finally get:

$\log _{10}(\tau / d)=1.21_{-0.26}^{+0.49}$

or $16 \mathrm{~d}$ (1- $\sigma$ range between 9 and 50 days). Note that the distribution about the mean is very asymmetric which implies that the upper limit is quite tentative. Moreover, the quoted lifetime is very close to the bin width (resolution) in the power spectrum and the mean can thus be influenced by the observation time length. However, the lower limit of 9 days is quite secure and valuable.

An interesting work to test the accuracy of the lifetime would be to compare the lifetime deduced from the scatter of the $\ell=0$ and $\ell=2$ modes. Unlike $\ell=0$ modes, $\ell=2$ modes can be affected by the rotational splitting ( $v \sin i$ of $2.6 \mathrm{~km} \mathrm{~s}^{-1}$ determined from the CORALIE spectra) and their scatter should be larger. However, the number and the non-consecutive position at all of such modes (see Fig. 3) do not allow us to do it.

\subsection{Frequency accuracy}

Now that we have determined the mode lifetime, we can estimate the uncertainties in the mode frequencies. To do this, we made 850 additional simulations using several different amplitudes and assuming noise and sampling similar to the $v$ Indi series in order to determine the scatter of the detected modes. The result of the simulations allowed an estimate of the accuracy for the individual detected frequencies, including the effect of the mode lifetime, and these are given in Table 2.

\section{Conclusions}

Our observations of $v$ Indi from two sites have allowed us to identify 13 oscillation modes. The mode identification was complicated by the apparent effects of avoided crossings. We found a large and small separation of $\Delta v=25.14 \pm 0.09 \mu \mathrm{Hz}$ and $\delta v_{02}=2.96 \pm 0.22 \mu \mathrm{Hz}$ at $330 \mu \mathrm{Hz}$.

Based on the scatter of the observed frequencies, we inferred a mode lifetime of about 16 days (1- $\sigma$ range between 9 and 50 days). The lower limit of 9 days is secure whereas the mean and upper limits values can be influenced by the observation time length and have to be taken with caution. Note that the lifetime of $v$ Indi, even its lower limit of 9 days, is far greater than previous detections on other stars as the Sun (Chaplin et al. 1997), the dwarf stars $\alpha$ Cen A and B (Kjeldsen et al. 2005) and the giant $\xi$ Hya (Stello et al. 2006). This new measurement of damping time, which is until now a poorly known parameter, is very different from the previous ones and thus present a challenge to theoretical models. The theoretical study of $v$ Indi, with asteroseismic and non-asteroseismic constraints, is postponed to a third paper.

Acknowledgements. This work was supported financially by the Swiss National Science Foundation, the Australian Research Council, the Danish Natural Science Research Council, the Danish National Research Foundation through its establishment of the Theoretical Astrophysics Center, and by a research associate fellowship from Penn State University. We further acknowledge support by NSF grant AST-9988087 (RPB) and by SUN Microsystems.

\section{References}

Bedding, T. R., \& Kjeldsen, H. 2006, Mem. Soc. Astron. Ital., 77, 384

Bedding, T. R., \& Kjeldsen, H. 2007, SOHO 18/GONG 2006/HELAS conference, Beyond the Spherical Sun: a New Era of Helio- and Asteroseismology, ESA SP-624, 25

Bedding, T. R., Kjeldsen, H., Butler, R. P., et al. 2004, ApJ, 614, 380

Bedding, T. R., Butler, R. P., Carrier, F., et al. 2006, ApJ, 647, 558

Bouchy, F., \& Carrier, F. 2002, A\&A, 390, 205

Carrier, F., \& Bourban, G. 2003, A\&A, 406, L23

Carrier, F., Eggenberger, P., \& Bouchy, F. 2005a, A\&A, 434, 1085

Carrier, F., Eggenberger, P., D’Alessandro, A., \& Weber, L. 2005b, New Astron., 10,315

Chaplin, W. J., Elsworth, Y., Isaak, G. R., et al. 1997 MNRAS, 288, 623

Christensen-Dalsgaard, J., Bedding, T. R., \& Kjeldsen, H. 1995, ApJ, 443, L29

De Ridder, J., Arentoft, T., \& Kjeldsen, H. 2006, MNRAS, 365, 595

Fernandes, J., \& Monteiro, M. J. P. F. G. 2003, A\&A, 399, 243

Kjeldsen, H., Bedding, T. R., Baldry, I. K., et al. 2003, AJ, 126, 1483

Kjeldsen, H., Bedding, T. R., Butler, R. P., et al. 2005, ApJ, 635, 1281

Montgomery, M. H., \& O’Donoghue, D. 1999, Delta Scuti Star Newsletter, Vienna, 13, 28

Stello, D., Kjeldsen, H, Bedding, T. R., \& Buzasi, D. 2006, A\&A, 448, 709

Tassoul, M. 1980, ApJS, 43, 469

Toutain, T., \& Fröhlich, C. 1992, A\&A, 257, 287 